\title{
Synergistic effects of sulbactam in multi-drug-resistant Acinetobacter baumannii
}

\author{
Fatih Temocin, Fatma Sebnem Erdinc, Necla Tulek, Meryem Demirelli, \\ Gunay Ertem, Sami Kinikli, Eda Koksal
}

Infectious Diseases and Clinical Microbiology, Ankara Training and Research Hospital, Ankara, Turkey.

Submitted: February 3, 2014; Approved: May 2, 2015.

\begin{abstract}
Acinetobacter baumannii is a frequently isolated etiologic agent of nosocomial infections, especially in intensive care units. With the increase in multi-drug resistance of $A$. baumannii isolates, finding appropriate treatment alternatives for infections caused by these bacteria has become more difficult, and available alternate treatments include the use of older antibiotics such as colistin or a combination of antibiotics. The current study aimed to evaluate the in vitro efficacy of various antibiotic combinations against multi-drug resistant $A$. baumannii strains. Thirty multi-drug and carbapenem resistant A. baumannii strains isolated at the Ankara Training and Research Hospital between June 2011 and June 2012 were used in the study. Antibiotic susceptibility tests and species-level identification were performed using conventional methods and the VITEK 2 system. The effects of meropenem, ciprofloxacin, amikacin, tigecycline, and colistin alone and in combination with sulbactam against the isolates were studied using Etest (bioMérieux) in Mueller-Hinton agar medium. Fractional inhibitory concentration index (FIC) was used to determine the efficacy of the various combinations. While all combinations showed a predominant indifferent effect, a synergistic effect was also observed in 4 of the 5 combinations. Synergy was demonstrated in $43 \%$ of the isolates with the meropenem-sulbactam combination, in $27 \%$ of the isolates with tigecycline-sulbactam, and in $17 \%$ of the isolates with colistin-sulbactam and amikacin-sulbactam. No synergy was detected with the sulbactam-ciprofloxacin combination and antagonism was detected only in the sulbactamcolistin combination (6.66\% of the isolates). Antibiotic combinations can be used as an alternative treatment approach in multi-drug resistant $A$. baumannii infections.
\end{abstract}

Key words: acinetobacter, antimicrobial drugs, drug resistance, drug synergism.

\section{Introduction}

Acinetobacter species are prevalent agents of nosocomial infections (Bergogne-Berezin and Towner, 1996) as they are resistant to environmental conditions and are capable of easily acquiring resistance to antibiotics; and the most commonly isolated species is Acinetobacter baumannii (Roberts et al., 2001).

In the recent years, due to the increase in the use of wide-spectrum antibiotics, Acinetobacter species has acquired resistance against these antibiotics. This resistance causes serious problems in providing effective treatment, particularly in intensive care units where antibiotic use is high and interventional procedures (e.g., intubation and uri- nary and intravenous catheters) are frequently performed (Chastre, 2003).

Alternate treatment protocols are being researched as the rate of resistance is increasing. These alternates include regimens using combination of antibiotics and synthesis of new antibiotics. Antimicrobial drug combinations are used to achieve a wider spectrum; they prevent the emergence of drug-resistant mutants, minimize toxicity, and achieve a synergistic effect.

In vitro synergy tests are used to evaluate drug interaction in antibiotic combinations, and include the checkerboard, time-kill, and Etest diffusion methods (Moody, 2004; Haddad et al., 2005). 
The current study aimed to determine in vitro effects of a combination of sulbactam (SUL) with tigecycline (TGC), meropenem (MP), amikacin (AK), ciprofloxacin (CL), or colistin (CT) against multi-drug resistant nosocomial A. baumannii species.

\section{Materials and Methods}

Thirty multi-drug and carbapenem resistant $A$. baumannii species isolated from blood cultures of patients hospitalized in the Ankara Training and Research Hospital between June 2011 and June 2012 were included in the study. Antibiotic susceptibility testing and species-level identification were carried out using conventional methods and the VITEK 2 system (bioMérieux SA, France). Although no current standard definition exists for the term "multi-drug resistance" with respect to Acinetobacter sp., resistance to three or more antibiotic classes used in the treatment of Acinetobacter infections is currently accepted as multi-drug resistance. The isolates used in this study were obtained from different clinics and patients hospitalized on different dates, and only one clinical isolate from each patient was included. All isolates were stored at $-20^{\circ} \mathrm{C}$ in brain-heart medium (Oxoid, UK) containing glycerol until use.

The effects of meropenem, ciprofloxacin, amikacin, tigecycline, and colistin alone, and in combination with sulbactam, against the 30 isolates were studied using the Etest method (bioMérieux, France) in Mueller-Hinton agar medium (Oxoid, UK) according to the manufacturer's instructions. The susceptibility breakpoint of the antibiotics used against $A$. baumannii is shown in Table 1 (Henwood et al., 2002; Kahlmeter et al., 2006; CLSI, 2007).

The fractional inhibitory concentration (FIC) index was used to determine the efficacy of each combination (Sopirala et al., 2010). To determine the FIC index using the Etest, the minimum inhibitory concentration (MIC) of the two antibiotics (denoted as A or B) involved in the combination was first calculated and recorded. To establish the

Table 1 - CLSI (2007) MIC values of antimicrobials against $A$. baumannii strains

\begin{tabular}{lccc}
\hline \multirow{2}{*}{ Antibiotic } & \multicolumn{3}{c}{ MIC $(\mu \mathrm{g} / \mathrm{mL})$ breakpoints } \\
\cline { 2 - 4 } & Sensitive & Intermediate & Resistant \\
\hline Colistin & $\leq 2$ & & $\geq 4$ \\
Ciprofloxacin & $\leq 1$ & 2 & $\geq 4$ \\
Meropenem & $\leq 4$ & 8 & $\geq 16$ \\
Amikacin & $\leq 16$ & 32 & 64 \\
Tigecycline* & $\leq 2$ & $2-8$ & $\geq 8$ \\
Sulbactam** & $\leq 4$ & 8 & $\geq 16$ \\
\hline
\end{tabular}

*Standards determined by FDA for Enterobacteriaceae.

**Sulbactam values were used according to CLSI (2007) MIC breakpoints for ampicillin-sulbactam.
MIC value of the combination, the B strip was placed on the medium and incubated at $37^{\circ} \mathrm{C}$ for $1 \mathrm{~h}$. Then, the B strip was removed and the A strip was placed such that it completely overlapped the concentration lines of the B strip. After incubation at $37^{\circ} \mathrm{C}$ for $24 \mathrm{~h}$, the intersection of the inhibition zone diameter at the edge of the Etest band was recorded as the MIC value of A in the combination. The same procedure was repeated with each antibiotic and for all combinations. To determine the efficacy of the combination, the FIC index was calculated according to the following equation:

$$
\begin{aligned}
& \text { FICA }=\frac{\text { MIC of drug A in combination }}{\text { MIC of drug A alone }} \\
& \text { FIC B }=\frac{\text { MIC of drug B in combination }}{\text { MIC of drug B alone }} \\
& \Sigma \text { FIC index }=\text { FIC A+ FIC B. }
\end{aligned}
$$

The $\Sigma$ FIC index values were interpreted as follows:

$$
\begin{aligned}
& \Sigma \text { FIC index } \leq 0.5=\text { synergistic } \\
& \Sigma \text { FIC index }>0.5 \text { to } 1=\text { additive } \\
& \Sigma \text { FIC index }>1 \text { to }<4=\text { indifferent (ineffective) and } \\
& \Sigma \text { FIC } \geq 4=\text { antagonistic interaction }
\end{aligned}
$$

Pseudomonas aeruginosa American Type Culture Collection (ATCC) 27853 and Escherichia coli ATCC 25922 were used as control species.

\section{Results}

Table 2 shows the MIC values $(\mu \mathrm{g} / \mathrm{mL})$, MIC50 values, MIC90 values $(\mu \mathrm{g} / \mathrm{mL})$, and the susceptibility ratios of the multi-drug and meropenem-resistant isolates.

As shown in the Table 2, all of the isolates were resistant to meropenem and ciprofloxacin but susceptible to colistin. Resistance to amikacin (56\%) but susceptibility to tigecycline were also high (47\%).

A total of $150 \Sigma$ FIC values were calculated for the five antibiotic combinations (CT-SUL, MP-SUL, CL-SUL, AK-SUL, TGC-SUL) for all isolates. Our interpretation of the $\Sigma$ FIC values (synergistic, additive, ineffective (indifferent), and antagonistic) are shown in Table 3 , and data on the percentage of the interaction are presented in Table 4.

In the current study, for all drug combinations, the most common interaction observed was indifferent or additive. CL-SUL had the highest indifferent effect at $90 \%$ (27/30 isolates), while TGC-SUL had the highest additive effect at 43\% (13/30). MP-SUL showed the highest synergistic effect at $43 \%$ (13/30) followed by TGC-SUL at $27 \%$ $(8 / 30)$. Antagonistic interference was observed only in the CT-SUL combination ( $6.66 \%, 2 / 30$ isolates). 


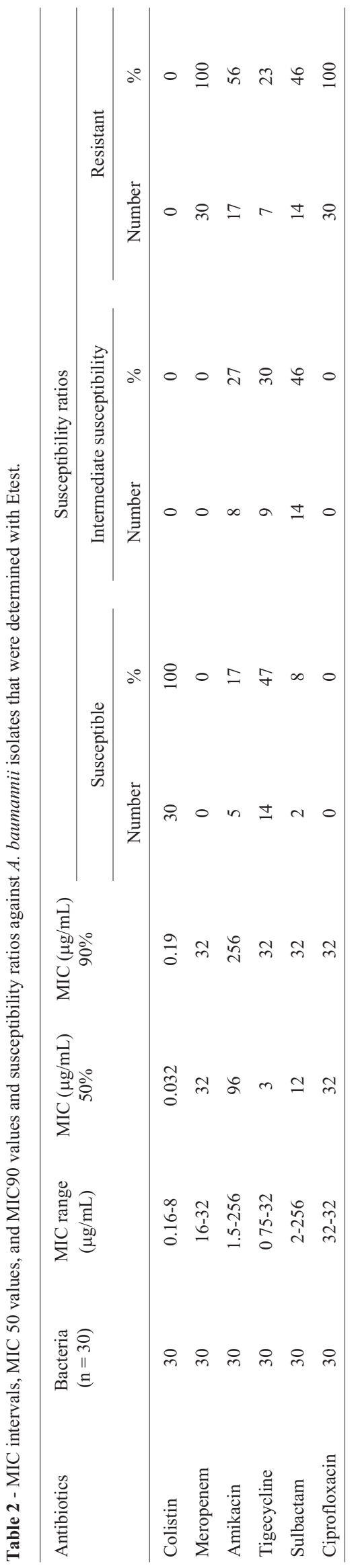

\section{Discussion}

The emergence of high antibiotic resistance in $A$. baumannii isolates has impeded the successful treatment of these infections, thus necessitating alternative treatment options. Among the available options, the use of a combination of antibiotics is currently the most preferred treatment approach (Falagas and Kasiakou, 2005).

Combination treatment is mainly used to prevent the development of antibiotic resistance and decrease dosedependent side effects, and to treat polymicrobial infections. Furthermore, it is also used to treat severe infections with high rates of mortality as a combination of antibiotics provides a synergistic effect against the multi-drug-resistant isolates (Kiffer et al., 2005).

While a combination of antimicrobial agents with different mechanisms of action may provide better pharmacokinetic effects or synergy, they may also cause antagonism. The absence of antagonistic interaction among antibiotics has clinical importance, and therefore, many studies have emphasized the need to determine the interactive effects of antibiotic combinations in vitro. A synergistic effect is especially beneficial (is especially beneficial (Fass et al., 1990; Haddad et al., 2005). It is known that the combined administration of carbapenem and aminoglycoside group of antibiotics, the most frequently used combination in the empiric treatment of Acinetobacter infections, generally demonstrates an in vitro synergistic effect (Marques et al., 1997; Bonapace et al., 2000). However, due to the prevalence of high resistance to both the groups of drugs, as shown in the present study and by previous reports in recent years, colistin appears to be the only viable treatment option. However, as colistin is associated with high nephrotoxicity and neurotoxicity current research is focused on using other treatment options, including use of different antibiotic and drug combinations (Falagas and Kasiakou, 2006; Dinc et al., 2013). Thus, the present study aimed to investigate the in vitro interactions between sulbactam and available antibiotics as possible treatment options.

Sulbactam alone has demonstrated direct antimicrobial activity against Bacteroides fragilis and Acinetobacter spp., and has intrinsic bactericidal activity against multidrug-resistant Acinetobacter spp., as it inhibits the penicillin-binding proteins (Allen \& Hartman 2010). Tazobactam and clavulanate are less effective compared to sulbactam, but there are no well-documented clinical practice guidelines. In the current study, the MIC50 and MIC90 values of sulbactam were found to be $12 \mu \mathrm{g} / \mathrm{mL}$ and $32 \mu \mathrm{g} / \mathrm{mL}$, respectively and these values are in agreement with previously reported values. Swenson et al. (2004) have evaluated the efficacy of sulbactam against 195 A. baumannii isolates and reported MIC50 and MIC90 values of $8 \mu \mathrm{g} / \mathrm{mL}$ and $128 \mu \mathrm{g} / \mathrm{mL}$, respectively, while Hawley et al. (2007) 
Table 3 - The list of FIC values for antibiotic combinations against multi-drug resistant $A$. baumannii isolates.

\begin{tabular}{|c|c|c|c|c|c|c|c|c|c|c|}
\hline \multirow[t]{2}{*}{$(\mathrm{n}=30)$} & \multicolumn{2}{|c|}{ CT-SUL } & \multicolumn{2}{|c|}{ MP-SUL } & \multicolumn{2}{|c|}{ CL-SUL } & \multicolumn{2}{|c|}{ AK-SUL } & \multicolumn{2}{|c|}{ TGC-SUL } \\
\hline & $\Sigma \mathrm{FIC}$ & Effect & $\Sigma$ FIC & Effect & $\Sigma$ FIC & Effect & $\Sigma$ FIC & Effect & $\Sigma$ FIC & Effect \\
\hline 1 & 0.0166 & $\mathrm{~S}$ & 0.13 & $\mathrm{~S}$ & 0.546 & ADD & 0.148 & S & 0.09 & $\mathrm{~S}$ \\
\hline 2 & 0.123 & S & 0.156 & $\mathrm{~S}$ & 0.697 & ADD & 0.156 & S & 0.421 & $\mathrm{~S}$ \\
\hline 3 & 0.124 & $\mathrm{~S}$ & 0.18 & $\mathrm{~S}$ & 0.843 & ADD & 0.421 & S & 0.5 & $\mathrm{~S}$ \\
\hline 4 & 0.251 & $\mathrm{~S}$ & 0.27 & $\mathrm{~S}$ & 1.011 & ID & 0.43 & S & 0.5 & $\mathrm{~S}$ \\
\hline 5 & 0.338 & $\mathrm{~S}$ & 0.281 & $\mathrm{~S}$ & 1.015 & ID & 0.5 & S & 0.5 & $\mathrm{~S}$ \\
\hline 6 & 1 & ID & 0.281 & $\mathrm{~S}$ & 1.023 & ID & 0.523 & ADD & 0.5 & $\mathrm{~S}$ \\
\hline 7 & 1.001 & ID & 0.39 & $\mathrm{~S}$ & 1.024 & ID & 0.523 & ADD & 0.5 & $\mathrm{~S}$ \\
\hline 8 & 1.005 & ID & 0.39 & $\mathrm{~S}$ & 1.062 & ID & 0.546 & ADD & 0.5 & $\mathrm{~S}$ \\
\hline 9 & 1.015 & ID & 0.401 & $\mathrm{~S}$ & 1.125 & ID & 0.546 & $\mathrm{ADD}$ & 0.56 & $\mathrm{ADD}$ \\
\hline 10 & 1.392 & ID & 0.406 & $\mathrm{~S}$ & 1.25 & ID & 0.593 & ADD & 0.59 & $\mathrm{ADD}$ \\
\hline 11 & 1.393 & ID & 0.421 & $\mathrm{~S}$ & 1.335 & ID & 0.729 & ADD & 0.593 & ADD \\
\hline 12 & 1.453 & ID & 0.468 & $\mathrm{~S}$ & 1.335 & ID & 0.781 & $\mathrm{ADD}$ & 0.625 & ADD \\
\hline 13 & 1.469 & ID & 0.5 & $\mathrm{~S}$ & 1.337 & ID & 0.781 & $\mathrm{ADD}$ & 0.625 & $\mathrm{ADD}$ \\
\hline 14 & 1.47 & ID & 0.729 & $\mathrm{ADD}$ & 1.337 & ID & 0.796 & ADD & 0.625 & ADD \\
\hline 15 & 1.48 & ID & 0.78 & ADD & 1.338 & ID & 0.843 & $\mathrm{ADD}$ & 0.687 & $\mathrm{ADD}$ \\
\hline 16 & 1.484 & ID & 0.796 & ADD & 1.364 & ID & 1.01 & ID & 0.729 & ADD \\
\hline 17 & 2 & ID & 0.843 & ADD & 1.502 & ID & 1.02 & ID & 0.833 & ADD \\
\hline 18 & 2 & ID & 0.875 & $\mathrm{ADD}$ & 1.505 & ID & 1.03 & ID & 0.84 & $\mathrm{ADD}$ \\
\hline 19 & 2 & ID & 1 & ID & 1.507 & ID & 1.031 & ID & 0.843 & ADD \\
\hline 20 & 2 & ID & 1.01 & ID & 1.511 & ID & 1.031 & ID & 0.87 & ADD \\
\hline 21 & 2 & ID & 1.03 & ID & 1.523 & ID & 1.05 & ID & 0.916 & $\mathrm{ADD}$ \\
\hline 22 & 2 & ID & 1.04 & ID & 1.625 & ID & 1.166 & ID & 1 & ID \\
\hline 23 & 2 & ID & 1.04 & ID & 2 & ID & 1.166 & ID & 1.031 & ID \\
\hline 24 & 2.01 & ID & 1.06 & ID & 2.003 & ID & 1.375 & ID & 1.056 & ID \\
\hline 25 & 2.95 & ID & 1.06 & ID & 2.005 & ID & 1.375 & ID & 1.125 & ID \\
\hline 26 & 2.985 & ID & 1.125 & ID & 2.009 & ID & 1.523 & ID & 1.25 & ID \\
\hline 27 & 3 & ID & 1.52 & ID & 2.031 & ID & 1.546 & ID & 1.341 & ID \\
\hline 28 & 3.91 & ID & 1.523 & ID & 2.039 & ID & 1.56 & ID & 1.5 & ID \\
\hline 29 & 5.7 & $\mathrm{AG}$ & 1.562 & ID & 2.062 & ID & 2.04 & ID & 1.5 & ID \\
\hline 30 & 7.813 & $\mathrm{AG}$ & 2.02 & ID & 2500 & ID & 2.729 & ID & 1.666 & ID \\
\hline
\end{tabular}

CT: Colistin; MP: Meropenem; AK: Amikacin; TGC: Tigecycline; SUL: Sulbactam; CL: Ciprofloxacin; S: Synergistic; ADD: Additive; ID: Indifferent; AG: Antagonist; $\Sigma$ FIC: Fractional inhibitory concentration index.

have reported MIC50 and MIC90 values of $16 \mu \mathrm{g} / \mathrm{mL}$ and $64 \mu \mathrm{g} / \mathrm{mL}$, respectively.

A synergistic effect against $A$. baumannii species was observed when sulbactam was combined with ampicillin, carbapenem, or cefoperazone (Chu et al., 2013). Pongpech et al. (2010) also reported synergistic effects when a combination of meropenem-sulbactam $(70 \%)$ or colistin-sulbactam $(53 \%)$ was used against carbapenem, and multi-drug- resistant A. baumannii isolates. While Kiffer et al. (2005) reported $29 \%$ synergy (14/48 isolates) and $58.4 \%$ additivity (28/48 isolates) with a sulbactam-meropenem combination against MDR A. baumannii isolates using the CB method, conversely, Santimaleeworagun et al. (2011), using the same method, reported no synergistic interaction when a combination of sulbactam and colistin. We also demonstrated considerable synergistic effects when sulbactam 
Table 4 - Interpretation for interaction results of antibiotic combinations against multi-drug resistant A. baumannii isolates.

\begin{tabular}{|c|c|c|c|c|c|c|c|c|}
\hline \multirow[t]{3}{*}{ Combinations } & \multicolumn{8}{|c|}{ Observed effect } \\
\hline & \multicolumn{2}{|c|}{ Synergistic } & \multicolumn{2}{|c|}{ Additive } & \multicolumn{2}{|c|}{ Indifferent } & \multicolumn{2}{|c|}{ Antagonist } \\
\hline & Number & $\%$ & Number & $\%$ & Number & $\%$ & Number & $\%$ \\
\hline CT-SUL & 5 & 17 & 0 & 0 & 23 & 76 & 2 & 7 \\
\hline MP-SUL & 13 & 43 & 5 & 17 & 12 & 40 & 0 & 0 \\
\hline AK-SUL & 5 & 17 & 10 & 33 & 15 & 50 & 0 & 0 \\
\hline TGC-SUL & 8 & 27 & 13 & 43 & 9 & 30 & 0 & 0 \\
\hline
\end{tabular}

CT: Colistin; MP: Meropenem; AK: Amikacin; TGC: Tigecycline; SUL: Sulbactam; CL: Ciprofloxacin.

was combined with meropenem $(43 \%, 13 / 30)$, tigecycline $(27 \%, 8 / 30)$, colistin $(17 \%, 5 / 30)$, or amikacin $(17 \%, 5 / 30)$, but no synergistic interaction was observed with ciprofloxacin. Importantly, to the best of our knowledge, this is the first report on a synergistic effect between tigecycline and sulbactam, and this result warrants further investigation.

Although Santimaleeworagun et al. (2011) reported no antagonism with a combination of sulbactam and colistin, we observed a small antagonistic effect $(6.66 \%$ $(2 / 30)$ which prompted a reconsideration of the usefulness of this combination and its subsequent rejection.

Although there are several methods for detecting in vitro interactions between combinations of antibiotics, but none is standard. Synergy testing by Etest is an easy-toperform method that does not obscure the effects of the active drug when used in combination with other drugs (Sopirala et al., 2010). Even though we used sulbactam as the main antibiotic in the Etest, it is not possible to comment on the exact effect of sulbactam as we obtained different results with the various antibiotics tested. While the findings reported here demonstrate similarities with many previous studies in the literature, some differences also exist which could be due to the differences in methodology or resistance patterns of the bacteria tested.

Given the possibility of antibiotic resistance patterns being different for different isolates of $A$. baumannii, any effect observed with a given combination is expected to be strain-specific. This implies that synergy testing for various combinations of antibiotics should be carried out against each patient-based isolate of MDR A. baumannii.

Furthermore, as in vitro studies do not accurately represent in vivo conditions, the data obtained from such in vitro studies should be supported by similar results from adequately controlled clinical studies.

\section{References}

Allen DM, Hartman BJ (2010) Acinetobacter species. In: Mandel GL, Bennet JE, Dolin R (eds) Mandell, Douglas, and Bennett's Principles and Practice of Infectious Diseases.7 th ed.Churchill Livingstone, Philadelphia, pp 2881-2885.
Bergogne-Berezin E, Towner KJ (1996) Acinetobacter spp. as nosocomial pathogens: microbiological, clinical, and epidemiological features. Clin Microbiol Rev 9:148-165.

Bonapace CR, White RL, Friedrich LV et al. (2000) Evaluation of antibiotic synergy against Acinetobacter baumannii: a comparison with Etest, time-kill, and checkerboard methods. Diagn Microbiol Infect Dis 38:43-50.

Chastre J (2003) Infections due to Acinetobacter baumannii in the ICU. Semin Respir Crit Care Med 24:69-78.

Chu H, Zhao L, Wang M et al. (2013) Sulbactam-based therapy for Acinetobacter baumannii infection: a systematic review and meta-analysis. Brazilian J Infect Dis 17:389-394.

Clinical and Laboratory Standards Institute (2007) Performance Standarts for Antimicrobial Susceptibility Testing; Seventeenth Informational Supplement. CLSI document M100-S17. CLSI, Wayne, pp 40-41.

Dinc G, Demiraslan H, Elmali F et al. (2013) Efficacy of sulbactam and its combination with imipenem, colistin and tigecycline in an experimental model of carbapenem-resistant Acinetobacter baumannii sepsis. Chemotherapy 59:325-329.

Falagas ME, Kasiakou SK (2005) Colistin: the revival of polymyxins for the management of multidrug-resistant gramnegative bacterial infections. Clin Infect Dis 40:1333-1341.

Falagas ME, Kasiakou SK (2006) Toxicity of polymyxins: a systematic review of the evidence from old and recent studies. Crit Care 10:R27.

Fass RJ, Gregory WW, D’Amato RF et al. (1990) In vitro activities of cefoperazone and sulbactam singly and in combination against cefoperazone-resistant members of the family Enterobacteriaceae and nonfermenters. Antimicrob Agents Chemother 34:2256-2259.

Haddad FA, Van Horn K, Carbonaro C et al. (2005) Evaluation of antibiotic combinations against multidrug-resistant Acinetobacter baumannii using the E-test. Eur J Clin Microbiol Infect Dis 24:577-579.

Hawley JS, Murray CK, Griffith ME et al. (2007) Susceptibility of acinetobacter strains isolated from deployed U.S. military personnel. Antimicrob Agents Chemother 51:376-378.

Henwood CJ, Gatward T, Warner M et al. (2002) Antibiotic resistance among clinical isolates of Acinetobacter in the UK, and in vitro evaluation of tigecycline (GAR-936). J Antimicrob Chemother 49:479-487.

Kahlmeter G, Brown DF, Goldstein FW et al. (2006) European Committee on Antimicrobial Susceptibility Testing 
(EUCAST) Technical Notes on antimicrobial susceptibility testing. Clin Microbiol Infect 12:501-503.

Kiffer CR, Sampaio JL, Sinto S et al. (2005) In vitro synergy test of meropenem and sulbactam against clinical isolates of Acinetobacter baumannii. Diagn Microbiol Infect Dis 52:317-322.

Marques MB, Brookings ES, Moser SA et al. (1997) Comparative in vitro antimicrobial susceptibilities of nosocomial isolates of Acinetobacter baumannii and synergistic activities of nine antimicrobial combinations. Antimicrob Agents Chemother 41:881-885.

Moody J (2004) Susceptibility tests to evaluate synergism. In: Isenberg, H.D. (ed) Clinical Microbiology Procedures Handbook. ASM Press, Washington DC, 5.12.1-5.12.23.

Pongpech P, Amornnopparattanakul S, Panapakdee S et al. (2010) Antibacterial activity of carbapenem-based combinations againts multidrug-resistant Acinetobacter baumannii. J Med Assoc Thai 93:161-171.
Roberts SA, Findlay R, Lang SD (2001) Investigation of an outbreak of multi-drug resistant Acinetobacter baumannii in an intensive care burns unit. J Hosp Infect 48:228-232.

Santimaleeworagun W, Wongpoowarak $\mathrm{P}$, Chayakul $\mathrm{P}$ et al. (2011) In vitro activity of colistin or sulbactam in combination with fosfomycin or imipenem against clinical isolates of carbapenem-resistant Acinetobacter baumannii producing OXA-23 carbapenemases. Southeast Asian J Trop Med Public Health 42:890-900.

Sopirala MM, Mangino JE, Gebreyes WA et al. (2010) Synergy testing by Etest, microdilution checkerboard, and time-kill methods for pan-drug-resistant Acinetobacter baumannii. Antimicrob Agents Chemother 54:4678-4683.

Swenson JM, Killgore GE, Tenover FC (2004) Antimicrobial susceptibility testing of Acinetobacter spp. by NCCLS broth microdilution and disk diffusion methods. J Clin Microbiol 42:5102-5108.

$$
\text { Associate Editor: Roxane Maria Fontes Piazza }
$$

All the content of the journal, except where otherwise noted, is licensed under a Creative Commons License CC BY-NC. 